\title{
Over-the-scope clipping and detachable snare ligation as a novel combination hemostatic therapy for diverticular bleeding
}

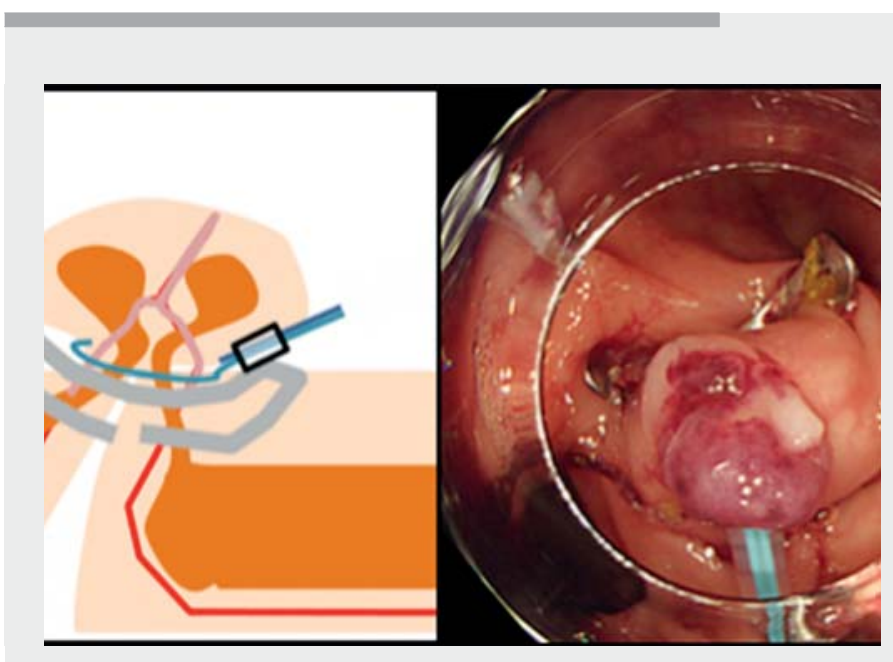

Video 1 The combination method of over-the-scope clipping and detachable snare ligation as a novel hemostatic treatment for colonic diverticular bleeding and prevention of rebleeding and perforation.

Ligation therapy, such as endoscopic band ligation and detachable snare ligation, is an effective treatment for colonic diverticular hemorrhage $[1,2]$. However, delayed perforation has been reported in patients undergoing steroid therapy and endoscopic ligation [3]. As an alternative, over-the-scope (OTS) clipping enables full-thickness resection at the central tissue mound of the inverted diverticulum while preventing perforation ( $\mathbf{F i g} \mathbf{1}$ ) [4]. Recent reports have described that OTS clipping is effective for hemostasis of diverticular bleeding; however, early rebleeding was observed in $33 \%$ of patients treated with OTS clips alone ( Fig. 2a, > Fig. 3) [5].

We developed a combination method of OTS clipping and detachable snare ligation as a novel treatment for diverticular bleeding to reduce the occurrence of rebleeding and delayed perforation ( Fig. 2 b-d, $>$ Video 1).

A 79-year-old man taking $15 \mathrm{mg}$ prednisolone daily for remitting seronegative symmetrical synovitis with pitting edema (RS3PE) syndrome and low-dose aspirin for angina pectoris presented with hema-

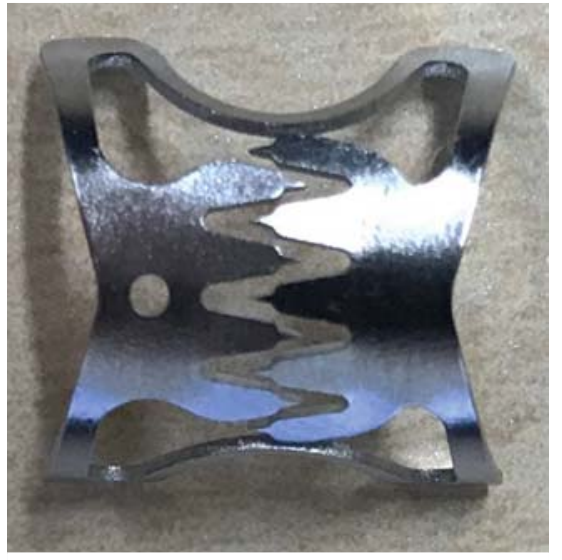

Fig. 1 An over-the-scope clip.

tochezia. Colonoscopy revealed diverticular bleeding in the ascending colon ( Fig.4). Standard marking clips were placed near the diverticulum. The scope, mounted with the OTS clipping system, was then inserted and the clip was released onto the diverticulum, allowing primary hemostasis. The resulting central tissue mound of the inverted and bulged diverticulum was further ligated using a detachable snare to prevent rebleeding

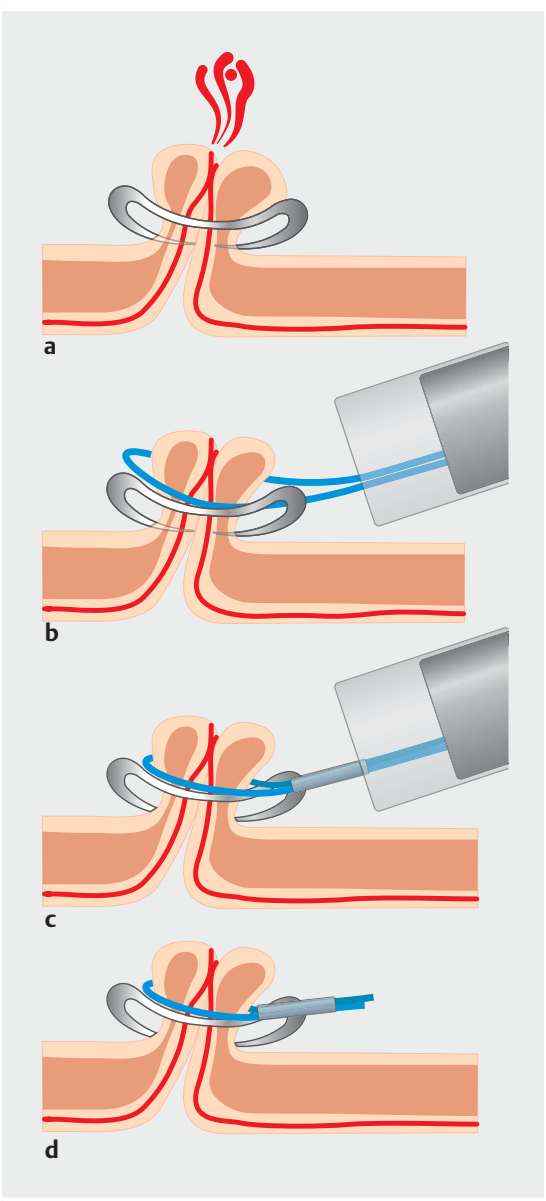

- Fig. 2 Schema of over-the-scope (OTS) clipping and snare ligation combination method. a Early rebleeding after OTS clip placement for hemostasis of colonic diverticular hemorrhage. $\mathbf{b}-\mathbf{d}$ The OTS clip was released onto the diverticulum, allowing primary hemostasis. A detachable snare was then applied to ligate the central tissue mound of the inverted and bulged diverticulum to prevent rebleeding.

( $\downarrow$ Fig.5). The patient was discharged 6 days after endoscopic treatment without any adverse events or early rebleeding. We believe that the novel combination method described here is a safe and effective standard therapy for hemostasis of colonic diverticular hemorrhage, especially in patients with increased risk for perforation and rebleeding. 


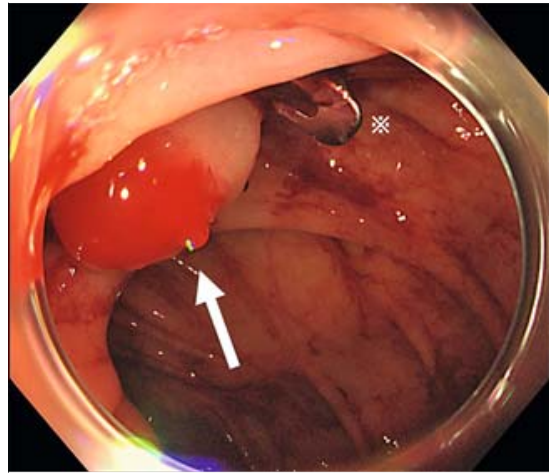

> Fig. 3 Early rebleeding after over-thescope clip $\left({ }^{*}\right)$ placement for hemostasis of colonic diverticular hemorrhage (arrow).

Endoscopy_UCTN_Code_TTT_1AQ_2AZ

Competing interests

The authors declare that they have no conflict of interest.

The authors

Koichiro Kawano', Reiko Kawano', Tomonori Moriguchi ${ }^{1}$, Hiroshi Tanabe', Takao Katoh', Katsuhisa Nishi ${ }^{1}$, Mamoru Takenaka ${ }^{2} \odot$

1 Department of Gastroenterology, Hyogo Prefectural Awaji Medical Center, Sumoto, Japan

2 Department of Gastroenterology and Hepatology, Kindai University Faculty of Medicine, Osaka-Sayama, Japan

\section{Corresponding author}

\section{Mamoru Takenaka, MD, PhD}

Department of Gastroenterology and Hepatology, Kindai University Faculty of Medicine, 377-2 Ohno-Higashi, OsakaSayama, 589-8511, Japan mamoxyo45@gmail.com

\section{References}

[1] Setoyama T, Ishii N, Fujita Y. Endoscopic band ligation (EBL) is superior to endoscopic clipping for the treatment of colonic diverticular hemorrhage. Surg Endosc 2011; 25 : 3574-3578

[2] Akutsu D, Narasaka T, Wakayama M et al. Endoscopic detachable snare ligation: a new treatment method for colonic diverticular hemorrhage. Endoscopy 2015; 47: 10391042
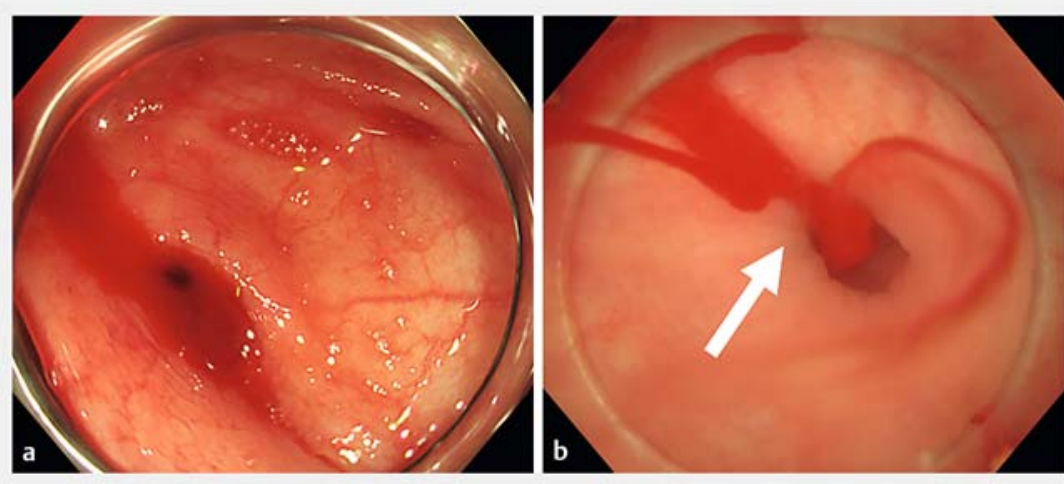

Fig. 4 a, b Active bleeding (arrow) from a diverticulum at the ascending colon.
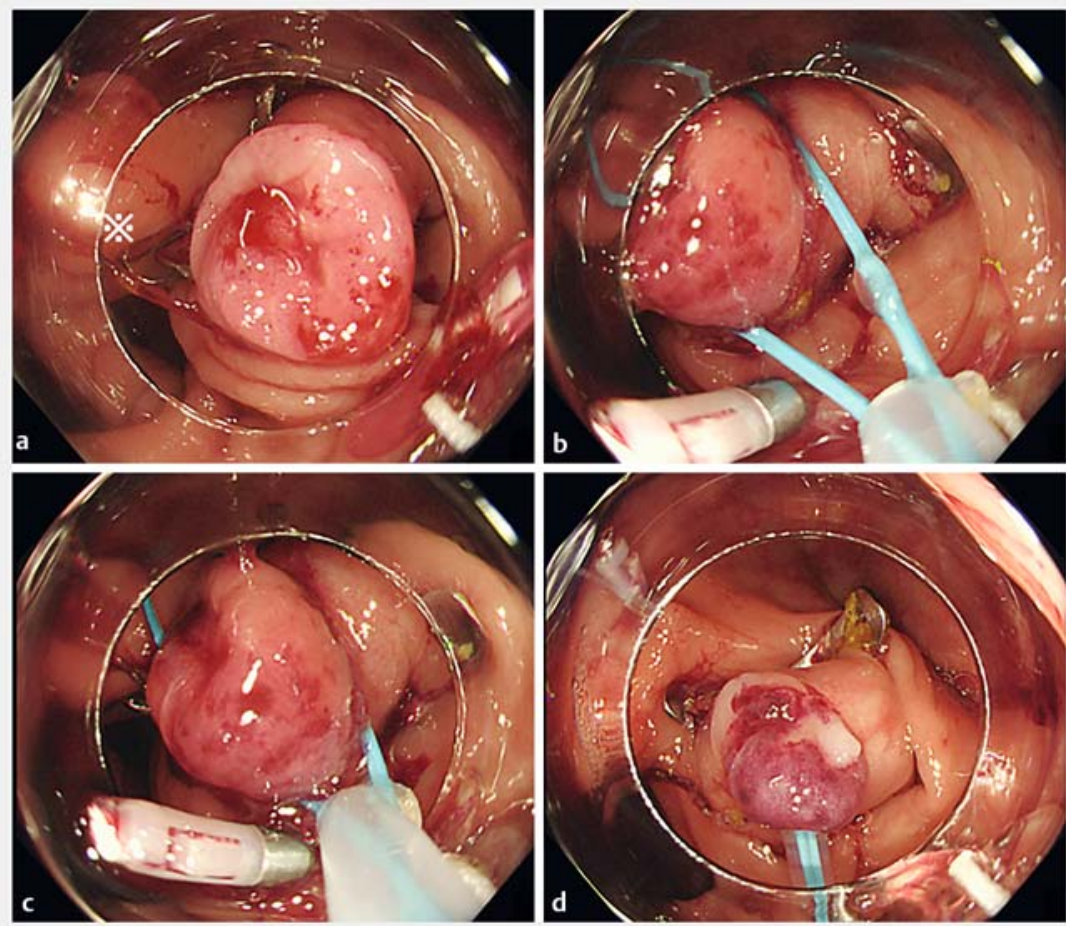

- Fig. 5 In addition to the primary hemostasis by over-the-scope clip (a), a detachable snare was applied to ligate the central tissue mound of the inverted and bulged diverticulum to prevent rebleeding $(\mathbf{b}-\mathbf{d})$.

[3] Takahashi S, Inaba T, Tanaka N. Delayed perforation after endoscopic band ligation for treatment of colonic diverticular bleeding. Dig Endosc 2016; 28: 484

[4] Agarwal A, Fang S, Pezhouh MK et al. Fullthickness resection of a rectal scar using a modified over-the-scope clip after piecemeal resection of intramucosal cancer. Endoscopy 2017; 49: E151-E152

[5] Wedi E, von Renteln D, Jung C et al. Treatment of acute colonic diverticular bleeding in high risk patients, using an over-thescope clip: a case series. Endoscopy 2016; 48: E383-E385

\section{Bibliography}

Endoscopy 2022; 54: 218-219

DOI 10.1055/a-1352-2162

ISSN 0013-726X

published online 5.3.2021

(c) 2021. Thieme. All rights reserved.

Georg Thieme Verlag KG, Rüdigerstraße 14,

70469 Stuttgart, Germany 